\title{
Consumo de tabaco en población escolarizada y su relación con los trastornos de la conducta alimentaria
}

\section{Tobacco smoking in school population and its relationship with eating disorders}

\author{
Josefa Castro Barea* y Serafina Castro Zamudio** \\ * Servicio Andaluz de Salud. Consejería de Salud y Bienestar Social de la Junta de Andalucía, España \\ **Departamento de Personalidad, Evaluación y Tratamiento Psicológico. Facultad de Psicología. Universidad de \\ Málaga, España
}

\section{Resumen}

Hay estudios que avalan una relación significativa entre el riesgo de padecer un Trastorno de la Conducta Alimentaria (TCA) y el consumo de tabaco como medida de control del apetito. El objetivo de este estudio es analizar la asociación entre el consumo de tabaco y actitudes y comportamientos presentes en la anorexia y bulimia nerviosas. El diseño del estudio es observacional analítico de casos y controles. La muestra estuvo constituida por 300 participantes de ambos sexos, de entre 12 y 20 años de edad, que respondieron a los siguientes instrumentos de evaluación: EAT-26, EDI-II, BULIT-R y un cuestionario adaptado sobre comportamientos, actitudes y opiniones hacia el tabaco. Se categorizaron las variables cuantitativas continuas y se agruparon a los participantes en grupos sintomáticos y asintomáticos. En el grupo sintomático, se constató que en la variable "haber fumado diariamente, al menos un cigarrillo, durante seis 0 más meses en la vida" se obtuvo una diferencia estadísticamente significativa $\chi^{2}(1, N=300)=7.536, p<.01$, y de magnitud baja ( $V=.159)$, en el EAT-26. Para el BULIT-R en esta misma variable se alcanzó una diferencia estadísticamente significativa $\chi^{2}(1, N=300)=8.740, p<.01$, y de magnitud baja $(\mathrm{V}=.171)$. Para la variable "consumo diario actual de cigarrillos" se alcanzó una diferencia estadísticamente significativa $\chi^{2}(2, N=300)=15.561, p<.01$, y de magnitud baja ( $V=.228)$, en el EAT-26. Para el BULIT-R en esta misma variable se obtuvo una diferencia estadísticamente significativa $\chi^{2}(2, N=300)=6.237, p<.05, y$ de magnitud baja $(\mathrm{V}=.144)$. Los resultados constatan la relación existente entre el consumo de tabaco y grupos sintomáticos afines a los TCA.
\end{abstract}

Palabras clave: consumo de tabaco, adolescente, anorexia nerviosa, bulimia nerviosa, trastornos de la conducta alimentaria.

\begin{abstract}
Recent studies have found a significant association between the risk of developing an eating disorder and tobacco consumption as an appetite suppressant. The aim of this study was to determine the association between tobacco consumption and attitudes and behaviours associated with eating disorders. The study design was an analytical observational case-control study. The study included 300 participants of both genders participated (age range, 12--20 years). The following assessment questionnaires were used: EAT-26, EDI-II, BULIT-R, and an adapted questionnaire on behaviour, attitude, and opinions regarding tobacco. Continuous variables were categorized. Participants were grouped as symptomatic and asymptomatic. In the symptomatic group, there was a statistically significant difference between the variable "having smoked at least one cigarette daily for 6 or more months lifetime" and EAT-26 $\left(\chi^{2}=7.536,1 \mathrm{df}\right.$, Cramer's $\left.V=0.159, p<0.01\right)$ and BULIT-R $\left(\chi^{2}=8.740,1 \mathrm{df}\right.$, Cramer's V $=0.171, p<$ $0.01)$ scores. Similarly, there was a significant difference between the variable "current daily cigarette consumption" and the EAT-26 $\left(\chi^{2}=15.565,2 \mathrm{df}\right.$, Cramer's $V=0.228$, $p<0.01)$ and BULIT-R $\left(\chi^{2}=6.237,2 \mathrm{df}\right.$, Cramer's $V=0.144$, $p<0.05)$ scores. The results demonstrate an association between tobacco consumption and eating disorder spectrum symptoms.
\end{abstract}

Key words: tobacco smoking, adolescent, anorexia nervosa, bulimia nervosa, eating disorders.

Cómo citar este artículo: Castro, J., y Castro, S. (2017). Consumo de tabaco en población escolarizada y su relación con los trastornos de la conducta alimentaria. Escritos de Psicología, 10, 41-50.

Correspondencia: Serafina Castro Zamudio. Universidad de Málaga. Facultad de Psicología. Departamento de Personalidad, Evaluación y Tratamiento Psicológico. Campus de Teatinos s/n. 29071. Málaga. España E-mail: scastro@uma.es. E-mail coautora Josefa Castro Barea: jcastrobarea@yahoo.es 


\section{Introducción}

La adolescencia es el periodo que comprende la transición de la infancia a la vida adulta, durante la cual se producen cambios importantes en la composición corporal. La alimentación del adolescente debe favorecer un adecuado crecimiento y desarrollo y promover hábitos de vida saludables para prevenir trastornos nutricionales. Puede aparecer una preocupación excesiva por la imagen corporal, basándose en un determinado ideal de belleza, iniciándose así los regímenes para adelgazar que conducen a una ingesta insuficiente de muchos nutrientes, sobre todo en mujeres. Además, estas personas incrementan la actividad física o incluso inician conductas purgativas para el mantenimiento del peso. Existe el riesgo de que esta práctica conduzca a un verdadero TCA (Marugán, Monasterio y Pavón, 2010). El estudio de Bell, Lawton y Dittmar (2007) pone en evidencia el papel de los factores sociales en la insatisfacción corporal, pues ofrecen influyentes mensajes acerca de lo aceptable o inaceptable de ciertos atributos físicos.

Disponemos de datos cada vez más fiables sobre la incidencia real de estos trastornos en población española (Grupo de trabajo de la Guía de Práctica Clínica sobre Trastornos de la Conducta Alimentaria, 2009; Peláez Fernández, Raich Escursell y Labrador Encinas, 2010; Rivas, Bersabé y Castro, 2001; Toro, 2000), cuyos resultados apuntan a que el $4-5 \%$ de las mujeres jóvenes y adolescentes desarrollan algún trastorno de este tipo y, en torno al 1-3\% en población adolescente y joven de ambos sexos. Estos datos son muy similares a los obtenidos en otros países desarrollados, según apunta Peláez Fernández et al. (2010). Respecto a los datos de prevalencia, éstos pueden oscilar entre el 0.11 y el $0.64 \%$ para la anorexia nerviosa (AN) y del 1.24 al $2.29 \%$ para la bulimia nerviosa (BN) (Peláez, 2003; Peláez, Labrador y Raich, 2005).

Estos resultados vienen a corroborar los presentados en la Guía de Práctica Clínica sobre Trastornos de la Conducta Alimentaria del Sistema Nacional de Salud (Grupo de trabajo de la Guía de Práctica Clínica sobre Trastornos de la Conducta Alimentaria, 2009), donde se realiza una revisión de los estudios presentados en España y se concluye que la población de mayor riesgo comprende a mujeres en la franja de edad de 12 a 21 años, y se obtiene una prevalencia del $0.14 \%$ al $0.9 \%$ para la AN, del $0.41 \%$ al $2.9 \%$ para la $\mathrm{BN}$ y del $2.76 \%$ al $5.3 \%$ en el caso de los trastornos de la conducta alimentaria no especificados (TCANE). En total, se presentan unas cifras de prevalencia de los TCA del $4.1 \%$ al $6.41 \%$. En el caso de varones adolescentes, aunque existen menos estudios, la prevalencia sería del $0 \%$ para la AN, del $0 \%$ al $0.36 \%$ para la BN y del $0.18 \%$ al $0.77 \%$ para los TCANE, con una prevalencia total de 0.27 a 0.90 .

Se considera que los TCA tienen una etiología policausal interactuando de una forma compleja factores predisponentes, desencadenantes y mantenedores de la conducta problema (Garner y Garfinkel, 1980; Toro y Vilardell, 1987). Como señalan Castro-Fornieles et al. (2010), los problemas del abuso de sustancias en adolescentes con TCA se correlacionan con mayor número de problemas escolares, sociales y familiares que los que padeciendo un TCA no presentan consumo de sustancias, por lo que sería conveniente que se detectasen y se pudiera dar un enfoque terapéutico específico adecuándolo a estas personas. Para ello sería necesario distinguir entre los subgrupos de TCA y los diferentes tipos de sustancias, sin embargo son escasos los estudios que detallan el tipo de sustancia de uso o abuso que suele utilizar la población adolescente que cumple o que está en riesgo de cumplir alguno de los criterios diagnósticos del DSM-IV-TR para los TCA, y de los que existen, han mostrado una relación directa entre las dietas y los atracones (con o sin conductas purgativas) con el uso o abuso de drogas estimulantes, entre las que se encuentra el tabaco (Piran y Robinson, 2006; Stice y Shaw, 2003).

En esta línea, Bisetto Pons, Botella Guijarro y Sancho Muñoz (2012) encontraron relación entre los adolescentes que consumían drogas y situarse en alguno de los umbrales de riesgo de la escala de evaluación utilizada $\left[\chi^{2}(1, \mathrm{~N}=444)=3.87 ; \mathrm{p}=.49\right]$, no habiendo relación estadísticamente significativa con la variable "tipo de droga" (Alcohol, estimulantes, tabaco u otras). No obstante se apreciaba una relación significativa entre la variable "utilizar algún tipo de droga como supresora del apetito" y estar en riesgo de padecer un TCA, siendo el tabaco la droga más utilizada ( $66 \%$ de los englobados en el umbral de riesgo de la Eating Disorder Diagnotic Scale).

La prevalencia de fumadores diarios varía entre estudios, en varones oscila del 8.5 al $13.3 \%$ y en chicas del 12.7 al $16.4 \%$. Aunque se puede apreciar un patrón de descenso de la prevalencia de fumadores diarios adolescentes en España a partir de los diversos estudios existentes que ofrecen datos consistentes, hay que mantener la vigilancia debido a la existencia de oscilaciones (Mendoza Berjano, López Pérez y Sagrera, 2007; Villalbí et al., 2012).

Autores como Wiseman, Turco, Sunday y Halmi (1997) o Welch y Fairburn (1998) encontraron que las chicas diagnosticadas de BN era el grupo con mayor probabilidad de consumir tabaco y que tras un periodo de abstinencia eran las que tenían mayor probabilidad de recaer a causa de la preocupación por el peso. 
Stice y Shaw (2003)eating, and affective disturbances prospectively predicted onset of cigarette smoking in adolescent girls ( $\mathrm{N}=496$ estudiaron si la imagen corporal, comer y los problemas afectivos predecían el inicio del consumo de cigarrillos en chicas adolescentes $(n=496)$. Los resultados apuntaron a que una elevada insatisfacción corporal y patologías relacionadas con la ingesta de comida así como un elevado estado de ánimo negativo, se relacionaban significativamente con el inicio en el consumo de cigarrillos.

Anzengruber et al. (2006) encontraron que mujeres diagnosticadas con algún subtipo de TCA mostraban puntuaciones más altas en el consumo de tabaco y la dependencia a la nicotina que las del grupo control. De éstas, las mujeres diagnosticadas con algún subtipo de TCA caracterizado por presentar episodios de tipo purgativo o atracones, fueron las que presentaron puntaciones más altas. Los autores concluyeron que dada la alta prevalencia encontrada en el estudio, el impacto del tabaquismo en el mantenimiento de los síntomas relacionados con los TCA requiere de un abordaje específico, tanto en la investigación como en la intervención clínica.

El objetivo de este trabajo es estudiar la presencia y magnitud de la asociación entre el consumo de tabaco y actitudes y comportamientos afines a la AN y BN. Basándonos en la evidencia arrojada por los distintos autores en los estudios anteriormente descritos, se espera encontrar un mayor porcentaje de personas fumadoras en el grupo sintomático así como una fuerte asociación entre las variables objeto de estudio.

\section{Participantes}

\section{Método}

La muestra fue seleccionada mediante un muestreo intencional o de conveniencia y voluntaria, por ser una técnica de selección fácil, barata y accesible para las investigadoras. Estuvo compuesta por un total de 300 estudiantes de la provincia de Málaga (España), siendo el $45.33 \%$ chicos $(n=136)$ y el $54.66 \%$ chicas $(n=164)$, con edades comprendidas entre 12 y 20 años de edad $(M=14.13$ y $D T=2.02)$, escolarizados en tres centros educativos entre los niveles de $1^{\circ}$ de ESO (Enseñanza Secundaria Obligatoria), $2^{\circ}$ de Bachillerato y Ciclo Formativo.

\section{Procedimiento}

Una vez presentado y aprobado el estudio por parte de las direcciones de los tres Centros, desde cada una de las direcciones se envió una carta informativa a todos los padres o tutores legales solicitando el consentimiento informado para los alumnos menores de edad. Realizado dicho trámite, se procedió a la cumplimentación de los cuestionarios en el aula bajo la supervisión de la investigadora. La duración de la prueba osciló entre 50 y 75 minutos. El material se presentó en un único documento compuesto por los distintos cuestionarios, junto a un sobre en blanco para que el alumno pudiera introducirlo y cerrarlo una vez cumplimentado, garantizando con ello la confidencialidad de las respuestas. En todo momento se siguieron las normas éticas pertinentes.

\section{Instrumentos de evaluación}

Con objeto de medir las distintas variables relacionadas con el consumo de tabaco se utilizó el Cuestionario sobre comportamientos, actitudes y opiniones hacia el tabaco basado en las recomendaciones de la Organización Mundial de la Salud (Smart et al., 1980), adaptado por Becoña et al. (2000). Consta de 64 ítems con diferentes escalas de respuesta y cubre dos grandes áreas: comportamientos, actitudes y opiniones hacia el tabaco. Junto a estos dos aspectos básicos se incluyen preguntas acerca de la presión a favor del abandono del tabaco, probabilidad de abandonarlo, grado de aceptación de las normas para su control, opiniones sobre la nocividad del tabaco, razones para fumar o no fumar, normas sociales sobre consumo, etc. La ventaja que tiene utilizar este cuestionario es que permite comparar los resultados de distintos investigadores y de distintos países, así como reducir o incrementar el número de áreas o preguntas en función de aquel aspecto o aspectos que más nos interesen evaluar.

De otro lado, a fin de poder delimitar los casos y los controles así como estudiar la relación entre el consumo de tabaco y actitudes y comportamientos afines a los trastornos de la conducta alimentaria se utilizaron los siguientes Tests e Inventarios:

- Test de Actitudes Alimentarias (Eating Attitudes Test, EAT-26; Castro, Toro y Salamero, 1991; Garner et al., 1982). Es una escala de 26 ítems con 6 opciones de respuesta derivada de un análisis factorial del original EAT-40 (Garner y Garfinkel, 1979). El hecho de crear el EAT-26 se debió al intento por solucionar el escaso poder predictivo del EAT-40 entre los subtipos restrictivo y bulímico de la AN. En general, existe un acuerdo en que este cuestionario es útil para identificar problemas alimentarios en poblaciones no clínicas además de ser sensible a los cambios que se producen como resultado del 
tratamiento (Chiodo, 1985; Williamson, 1990). Se compone de tres subescalas: hacer dieta, bulimia y preocupación por la comida y control oral. El punto de corte se establece en 20 (King, 1989, 1991). La fiabilidad test-retest en un periodo de dos a tres semanas se sitúa en torno al $84 \%$. La sensibilidad y especificidad es del $95 \%$; el poder predictivo positivo es del $79 \%$ y del poder predictivo negativo del 94\% (Iñarritu, Cruz y Morán, 2004).

- Inventario de Trastornos de la Conducta Alimentaria (Eating Disorder Inventory, EDI-II; Corral, González, Pereña, y Seisdedos; 1998, adaptado por TEA; Garner, 1991). El EDI-II es un cuestionario de 91 ítems con 6 opciones de respuesta sobre síntomas normalmente asociados con la AN y BN; no pretende obtener en un diagnóstico específico sino en la observación y evaluación precisa de ciertos rasgos psicológicos o conjuntos de síntomas que se supone, tienen relevancia para comprender y poder tratar los TCA. Está dividido en dos partes clínicamente relevantes, tres subescalas de actitudes hacia la alimentación y ocho subescalas disfuncionales personales. Puntuaciones altas para cada una de las subescalas se correspondería con un nivel mayor de psicopatología relacionada con la alimentación. La fiabilidad en grupos sanos oscila entre .29 y .88, para grupos de riesgo entre .31 y .88 y para grupos clínicos entre .69 y .90 . Las escalas del EDI-II se han diseñado para medir rasgos independientes aunque algunos autores sugieren un punto de corte sensible de 80 (García, Vázquez, López y Arcila, 2003).

- Test de Bulimia Revisado (Bulimia Test Revised, BULIT-R; Berrios-Hernández et al., 2007; Thelen, Farmer, Wonderlich y Smith, 1991). Se trata de una versión revisada del test original que desarrollaron Smith y Thelen (1984), compuesto por 36 ítems con 5 opciones de respuesta, diseñado para evaluar los síntomas de la bulimia nerviosa basándose en los criterios diagnósticos del DSM-IV. En el análisis factorial se identificaron cinco dimensiones: atracones, control e imagen corporal, medidas radicales y ayuno, ejercicio físico, vómito y uso de laxantes y uso de diuréticos. La fiabilidad test-retest es del $95 \%$ con una alta consistencia interna (98\%) y una habilidad de falsos negativos del $80 \%$ y de falsos positivos del $40 \%$. La sensibilidad es del $91 \%$ y la especificidad del $96 \%$ con un poder predictivo positivo del $81 \%$ y negativo del $98 \%$ (Welch, Thompson y Hall, 1993). Se sugiere que los investigadores que utilicen este instrumento como prueba de tamizaje deberían utilizar un punto de corte de 85 para reducir el potencial de falsos negativos (Iñarritu et al., 2004).

\section{Análisis de los datos}

En primer lugar, con objeto de detectar valores atípicos y extremos, etiquetar los valores perdidos y/o no aplicables así como describir la distribución de cada variable, se realizó un análisis exploratorio de los datos mediante el paquete estadístico SPSS para Windows versión 15.0, calculando media aritmética, mediana, desviación estándar, mínimos y máximos para las variables cuantitativas y frecuencias absolutas y porcentajes para las cualitativas. De igual forma se calcularon las frecuencias absolutas y los porcentajes de la puntuación total obtenida por la muestra en los distintos instrumentos de evaluación utilizados para medir TCA a fin de poder definir y corroborar en la muestra estudiada los puntos de corte propuestos por los autores para cada instrumento de evaluación (Becoña, Palomares y García, 2000; Garner, Olmsted, Bohr y Garfinkel, 1982; Smart et al., 1980) ya que el tipo de diseño corresponde con un estudio observacional analítico de casos y controles, donde los casos, se refieren a aquellos participantes que han igualado o superado los puntos de corte, para cada instrumento de evaluación utilizado, y los controles, a aquellos participantes que no lo han superado.

Para estudiar la relación existente entre las puntuaciones totales obtenidas en el Test de Actitudes Alimentarias (EAT-26), el Inventario de Trastornos de la Conducta Alimentaria (EDI-II) y el Test de Bulimia Revisado (BULIT-R) con las variables "haber probado alguna vez un cigarrillo" y "haber fumado diariamente, al menos un cigarrillo, durante seis o más meses en la vida", al ser ambas variables cualitativas dicotómicas se llevó a cabo el análisis mediante la $U$ de Mann-Whitney. Para la variable "consumo actual de cigarrillos", al tratarse de una variable cualitativa politómica se utilizó la $H$ de Kruskal-Wallis.

A fin de seguir con el análisis de los datos, fue necesario realizar una categorización de las variables cuantitativas continuas y agrupar a los participantes en grupos sintomáticos (por encima del punto de corte) y asintomáticos (por debajo del punto de corte) para estudiar el comportamiento de aquellas variables relacionadas con el consumo de tabaco en grupos sintomáticos afines a la conducta alimentaria alterada y ver si dicho comportamiento se diferenciaba respecto del grupo asintomático. Para ello se prestó especial interés a los porcentajes, a los valores de Chi-cuadrado de Pearson, concretamente al grado de significación, así como al valor de la $V$ de Cramer, el cual nos indica la fuerza de dicha asociación. 


\section{Resultados}

De los 300 estudiantes incluidos en el estudio, el $26.7 \%$ refirió "haber probado alguna vez un cigarrillo", el $5.7 \%$ informó "haber fumado diariamente, al menos un cigarrillo, en los últimos seis meses o más meses en la vida" y el $3.7 \%$ mencionó tener un "consumo diario actual de cigarrillos" (tabla 1).

Tabla 1

Frecuencias y porcentajes de las variables relacionadas con el consumo de tabaco

\begin{tabular}{|c|c|c|c|c|}
\hline & & $\begin{array}{l}\text { No } \\
\mathrm{n}(\%)\end{array}$ & $\begin{array}{l}\text { Sí } \\
\mathrm{n}(\%)\end{array}$ & $\begin{array}{l}\text { Total } \\
\mathrm{N}(\%)\end{array}$ \\
\hline $\begin{array}{l}\text { Haber probado } \\
\text { alguna vez un cigarrillo }\end{array}$ & & $220(73.3 \%)$ & $80(26.7 \%)$ & $300(100 \%)$ \\
\hline \multirow{2}{*}{$\begin{array}{l}\text { Haber fumado diariamente, } \\
\text { al menos un cigarrillo, } \\
\text { durante seis o más meses } \\
\text { en la vida }\end{array}$} & & $283(94.3 \%)$ & $17(5.7 \%)$ & $300(100 \%)$ \\
\hline & $\begin{array}{l}\text { Nunca } \\
\mathrm{n}(\%)\end{array}$ & $\begin{array}{l}\text { A veces } \\
\mathrm{n}(\%)\end{array}$ & $\begin{array}{l}\text { A diario } \\
\mathrm{n}(\%)\end{array}$ & $\begin{array}{l}\text { Total } \\
\mathrm{N}(\%)\end{array}$ \\
\hline $\begin{array}{l}\text { Consumo diario actual de } \\
\text { cigarrillos }\end{array}$ & $281(93.7 \%)$ & $8(2.7 \%)$ & $11(3.7 \%)$ & $300(100 \%)$ \\
\hline
\end{tabular}

Nota. Ítems seleccionados del cuestionario sobre comportamientos, actitudes y opiniones hacia el tabaco, basado en las recomendaciones de la Organización Mundial de la Salud (Smart et al., 1980) y adaptado a nuestro medio (Becoña, Palomares y García, 2000).

Los resultados reflejan que $21(7 \%)$ participantes igualaron o superaron el punto de corte propuesto para el Test de Actitudes Alimentarias (EAT-26); 35 (11.7\%) lo hicieron para el Inventario de Trastornos de la Conducta Alimentaria y finalmente, $12(4 \%)$ fueron los participantes que superaron el punto de corte establecido en el Test de Bulimia Revisado. En las tablas 2 y 3 se muestran los estadísticos descriptivos de la puntuación total obtenida por la muestra en los instrumentos de evaluación utilizados para medir TCA.

Tabla 2

Estadísticos descriptivos de la puntuación total obtenida por la muestra en los distintos instrumentos de evaluación utilizados.

\begin{tabular}{llll}
\hline & EAT-26 & EDI-II & BULIT-R \\
\hline Media & 9.20 & 46.29 & 55.03 \\
Varón $(n=136)$ & 8.15 & 43.88 & 54.30 \\
Mujer $(n=164)$ & 10.06 & 48.28 & 55.63 \\
Media & 7 & 41 & 52 \\
Varón $(n=136)$ & 6 & 38.5 & 51 \\
Mujer $(n=164)$ & 8 & 42.5 & 53 \\
Media & 7.05 & 26.05 & 14.34 \\
Varón $(n=136)$ & 5.37 & 26.06 & 13.67 \\
Mujer $(n=164)$ & 8.10 & 25.96 & 14.89 \\
Media & 0 & 7 & 36 \\
Varón $(n=136)$ & 0 & 7 & 36 \\
Mujer $(n=164)$ & 0 & 10 & 37 \\
Media & 56 & 184 & 108 \\
Varón $(n=136)$ & 33 & 184 & 108 \\
Mujer $(n=164)$ & 56 & 152 & 108 \\
\hline Nota. EAT-26 & & 7 & 11, BULIT-R Bulimia Test
\end{tabular}

Nota. EAT-26= Eating Attitudes Test 26, EDI-II= Eating Disorder Inventory II, BULIT-R= Bulimia Test Revised. 
Tabla 3

Percentiles de las puntuaciones obtenidas por la muestra en los instrumentos utilizados para evaluar sintomatología relacionada con el comportamiento alimentario alterado.

\begin{tabular}{llllllll}
\hline & 5 & 10 & 25 & 50 & 75 & 90 & 95 \\
\hline EAT-26 & 2 & 3 & 5 & 7 & 12 & 17 & 21 \\
Varón $(n=136)$ & 2 & 3 & 5 & 6 & 11 & 17 & 18.15 \\
Mujer $(n=164)$ & 2 & 3 & 5 & 8 & 13 & 19.5 & 24 \\
EDI-II & 15 & 18 & 27 & 41 & 59 & 81 & 95 \\
Varón $(n=136)$ & 13 & 18 & 25 & 38.5 & 57 & 78.3 & 92.9 \\
Mujer $(n=164)$ & 15 & 19 & 29 & 42.5 & 60.75 & 88 & 96.5 \\
BULIT-R & 38 & 40 & 43 & 52 & 63 & 74 & 82.95 \\
Varón $(n=136)$ & 38 & 40.4 & 44 & 51 & 61 & 72.3 & 79.45 \\
Mujer $(n=164)$ & 38 & 40 & 43 & 53 & 64.75 & 78 & 85.5 \\
\hline
\end{tabular}

Nota. EAT-26= Eating Attitudes Test 26, EDI-II= Eating Disorder Inventory II, BULIT-R= Bulimia Test Revised.

Según la prueba $U$ de Mann-Whitney, en el BULIT-R, la variable "haber probado alguna vez un cigarrillo" produjo diferencias estadísticamente significativas $(U=6804 ; p<.01)$ al igual que la variable "haber fumado diariamente, al menos un cigarrillo, durante seis o más meses en la vida" ( $U=1236$; $\mathrm{p}<$.01). Por último, también se obtuvieron diferencias estadísticamente significativas en la prueba de Kruskal-Wallis $\left(X^{2}=9.79 ; p<.01\right)$ para la variable "consumo diario actual de cigarrillos" en el BULIT-R. Sin embargo, no se mostraron diferencias estadísticamente significativas en estas mismas variables y la puntuación total obtenida por los participantes en el EAT-26 y EDI-II (tabla 4).

Tabla 4

Relación entre consumo de tabaco y comportamiento alimentario alterado (Pruebas $U$ de Mann-Whitney y $\mathrm{H}$ de Kruskal-Wallis).

\begin{tabular}{|c|c|c|c|}
\hline EAT-26 & EDI-II & BULIT-R & \\
\hline \multicolumn{4}{|l|}{ Prueba U de Mann-Whitney } \\
\hline $\begin{array}{l}\text { Haber probado } \\
\text { alguna vez un cigarrillo } \\
\text { Si }(n=80) \\
\text { No }(n=220)\end{array}$ & 8655 & 8761.5 & $6804.5\left(^{* *}\right)$ \\
\hline $\begin{array}{l}\text { Haber fumado diariamente, } \\
\text { al menos un cigarrillo, durante } \\
\text { seis o más meses en la vida } \\
\text { Sí }(n=17) \\
\text { No }(n=283)\end{array}$ & 1910.5 & 1880 & $1236\left({ }^{* *}\right)$ \\
\hline \multicolumn{4}{|l|}{ Prueba $\mathrm{H}$ de Kruskal-Wallis } \\
\hline $\begin{array}{l}\text { Consumo diario actual de cigarrillos } \\
\text { Nunca }(n=281) \\
\text { A veces }(n=8) \\
\text { A diario }(n=11)\end{array}$ & 1.148 & 1.756 & $\left.9.7900^{* \star}\right)$ \\
\hline
\end{tabular}

En la tabla 5 se constata que en el EAT 26, el porcentaje de sujetos $8.75 \%(n=7)$ que se situaron por encima del punto de corte que decían "haber probado alguna vez un cigarrillo" o el porcentaje de sujetos $23.52 \%(n=4)$ que dijeron "haber fumado diariamente, al menos un cigarrillo, durante 6 o más meses en la vida" fue mayor que en el caso en que los sujetos respondieron con un "no" en ambas variables, cuyos porcentajes fueron $6.36 \%(n=14)$ y $6 \%(n=17)$ respectivamente. Esto mismo ocurrió en el EDI-II cuyos porcentajes en el grupo sintomático que afirmaron "haber probado alguna vez un cigarrillo" o "haber fumado diariamente, al menos un cigarrillo, durante seis o más meses en la vida" fueron, respectivamente, $13.75 \%(n=11)$ y $23.52 \%(n=4)$ frente al porcentaje de sujetos que dijeron no haberlo hecho, cuyos porcentajes fueron $10.9 \%(n=24)$ y $10.95(n=31)$. Por último, también en el BULIT-R el porcentaje de sujetos del grupo sintomático que respondieron de manera afirmativa fue mayor en ambas variables $6.25 \%(n=5)$ y $17.64 \%(n=3)$ que en el grupo sintomático que respondieron de manera negativa en ambas variables cuyos porcentajes fueron $3.18 \%(n=7)$ y $3.18 \%(n=9)$, respectivamente.

Por otro lado, en la variable "consumo diario actual de cigarrillos", el porcentaje de sujetos del grupo sintomático que dijeron fumar a diario y superaron el punto de corte en los 3 instrumentos de evaluación utilizados (EAT-26, EDI-II y BULIT-R) fue mayor $36.36 \%(n=4) ; 18.18 \%(n=2)$ y $18.18 \%(n=2)$ que el porcentaje de sujetos $6.04 \%(n=17), 11.74 \%(n=33)$ y $3.55 \%(n=10)$ que manifestaron no haber fumado nunca (tabla 5). 
Tabla 5

Tabla de contingencia consumo de tabaco y puntuación total EAT-26, EDI-Il y BULIT-R.

\begin{tabular}{|c|c|c|c|c|c|c|c|c|c|}
\hline & \multicolumn{3}{|c|}{ EAT-26 } & \multicolumn{3}{|c|}{ EDI-II } & \multicolumn{3}{|c|}{ BULIT-R } \\
\hline & GS & GA & Total & GS & GA & Total & GS & GA & Total \\
\hline \multicolumn{10}{|c|}{ Haber probado alguna vez un cigarrillo } \\
\hline Sí & 7 & 73 & 80 & 11 & 69 & 80 & 5 & 75 & 80 \\
\hline$\%$ & 8.75 & 91.25 & 100 & 13.75 & 86.25 & 100 & 6.25 & 93.75 & 100 \\
\hline No & 14 & 206 & 220 & 24 & 196 & 220 & 7 & 213 & 220 \\
\hline$\%$ & 6.36 & 93.63 & 100 & 10.90 & 89.09 & 100 & 3.18 & 96.81 & 100 \\
\hline Total & 21 & 279 & 300 & 35 & 265 & 300 & 12 & 288 & 300 \\
\hline$\%$ & 7 & 93 & 100 & 11.66 & 88.33 & 100 & 4 & 96 & 100 \\
\hline \multicolumn{10}{|c|}{ Haber fumado diariamente, al menos un cigarrillo, durante seis o más meses en la vida } \\
\hline Sí & 4 & 13 & 17 & 4 & 13 & 17 & 3 & 14 & 17 \\
\hline$\%$ & 23.52 & 76.47 & 100 & 23.52 & 76.47 & 100 & 17.64 & 82.35 & 100 \\
\hline No & 17 & 266 & 283 & 31 & 252 & 283 & 9 & 274 & 283 \\
\hline$\%$ & 6 & 93.99 & 100 & 10.95 & 89.04 & 100 & 3.18 & 96.81 & 100 \\
\hline Total & 21 & 279 & 300 & 35 & 265 & 300 & 12 & 265 & 300 \\
\hline$\%$ & 7 & 93 & 100 & 11.66 & 88.33 & 100 & 4 & 88.33 & 100 \\
\hline \multicolumn{10}{|c|}{ Consumo diario actual de cigarrillos } \\
\hline Nunca & 17 & 264 & 281 & 33 & 248 & 281 & 10 & 271 & 281 \\
\hline$\%$ & 6.04 & 93.95 & 100 & 11.74 & 88.25 & 100 & 3.55 & 96.44 & 100 \\
\hline A veces & 0 & 8 & 8 & 0 & 8 & 8 & 0 & 8 & 8 \\
\hline$\%$ & 0 & 100 & 100 & 0 & 100 & 100 & 0 & 100 & 100 \\
\hline A diario & 4 & 7 & 11 & 2 & 9 & 11 & 2 & 9 & 11 \\
\hline$\%$ & 36.36 & 63.63 & 100 & 18.18 & 81.81 & 100 & 18.18 & 81.81 & 100 \\
\hline Total & 21 & 279 & 300 & 35 & 265 & 300 & 12 & 288 & 300 \\
\hline$\%$ & 7 & 93 & 100 & 11.66 & 88.33 & 100 & 4 & 96 & 100 \\
\hline
\end{tabular}

Nota. EAT-26 = Eating Attitudes Test 26, EDI-II = Eating Disorder Inventory II, BULIT-R = Bulimia Test

Revised, GS = Grupo sintomático, GA = Grupo asintomático.

Finalmente, En el grupo sintomático, se constató que en la variable "haber fumado diariamente, al menos un cigarrillo, durante seis o más meses en la vida" se obtuvo una diferencia estadísticamente significativa $\chi^{2}(1, N=300)=7.536, p<.01$, y de magnitud baja $(V=.159)$, en el EAT-26. Para el BULIT-R en esta misma variable se alcanzó una diferencia estadísticamente significativa $\chi^{2}(1, N=300)=8.740$, $p<.01$, y de baja magnitud $(V=.171)$. Para la variable "consumo diario actual de cigarrillos" se alcanzó una diferencia estadísticamente significativa $\chi^{2}(2, N=300)=15.561, p<.01$, y de magnitud baja $(V=$ $0.228)$, en el EAT-26. Para el BULIT-R en esta misma variable se obtuvo una diferencia estadísticamente significativa $\chi^{2}(2, N=300)=6.237, p<.05$, y de baja magnitud $(V=.144)$. No se alcanzó nivel de significación en ninguna de las variables en el EDI-II (tabla 6).

Tabla 6

Test Chi-cuadrado y $V$ de Cramer (consumo de tabaco y puntuación total EAT-26, EDI-II y BULIT-R).

\begin{tabular}{|c|c|c|c|c|c|c|c|c|c|}
\hline & \multicolumn{3}{|c|}{ EAT-26 cat. } & \multicolumn{3}{|c|}{ EDI-II cat. } & \multicolumn{3}{|c|}{ BULIT-R cat. } \\
\hline & Valor & gl & Sig. Asint. & Valor & gl & Sig. Asint. & Valor & gl & Sig. Asint. \\
\hline \multicolumn{10}{|c|}{ Haber probado alguna vez un cigarrillo } \\
\hline $\begin{array}{l}\text { Chi.cuadrado } \\
\text { de Pearson }\end{array}$ & .513 & 1 & .474 & .459 & 1 & .498 & 1.438 & 1 & .230 \\
\hline V de Cramer & 041 & & & .041 & & & .069 & & \\
\hline \multicolumn{10}{|c|}{ Haber fumado diariamente, al menos un cigarrillo, durante seis o más meses en la vida } \\
\hline $\begin{array}{l}\text { Chi.cuadrado } \\
\text { de Pearson }\end{array}$ & 7.563 & 1 & $\left..0066^{* \star}\right)$ & 2.461 & 1 & 117 & 8.740 & 1 & $.003\left({ }^{* *}\right)$ \\
\hline V de Cramer & 159 & & & .091 & & & .171 & & \\
\hline \multicolumn{10}{|c|}{ Consumo diario actual de cigarrillos } \\
\hline $\begin{array}{l}\text { Chi.cuadrado } \\
\text { de Pearson }\end{array}$ & 15.561 & 2 & $.0000^{(*)}$ & 1.511 & 2 & .470 & 6.237 & 2 & $.044\left(^{*}\right)$ \\
\hline V de Cramer & .228 & & & .071 & & & . 144. & & \\
\hline
\end{tabular}

Nota. EAT-26 cat.= Eating Attitudes Test 26 categorizado, EDI-II cat. = Eating Disorder Inventory II categorizado, BULIT-R cat. $=$ Bulimia Test Revised categorizado,

${ }^{*} \mathrm{P}<.05$

${ }^{* *} P<.01$ 


\section{Discusión}

Los resultados encontrados en este estudio son congruentes con los hallados en otros estudios (Welch y Fairburn, 1998; Wiseman et al., 1997) donde se ha constatado una asociación positiva y significativa para todas las variables relacionadas con el consumo de tabaco y las puntuaciones totales obtenidas para la muestra en el Test de Bulimia Revisado (BULIT-R) pero dicha significación no se repite para esas mismas variables y la puntuación total obtenida por los participantes en el EAT-26 y EDI-II. De hecho, Wiseman et al. (1997) o Welch y Fairburn (1998), encontraron que las chicas diagnosticadas de BN era el grupo con mayor probabilidad de consumir tabaco, sin embargo a pesar de la alta distorsión en la imagen corporal, las adolescentes diagnosticadas de AN restrictiva no usaban el consumo de esta sustancia como estrategia para controlar el peso.

Este resultado se reafirma con posterioridad cuando evaluamos el comportamiento de estas mismas variables en el grupo sintomático y asintomático. Si atendemos al porcentaje de participantes que se sitúa por encima del punto de corte en cada test e inventario, los resultados apuntan en esta dirección. El porcentaje de participantes que dicen "haber probado alguna vez un cigarrillo" y "haber fumado diariamente, al menos un cigarrillo, durante 6 o más meses en la vida", es mayor dentro del grupo sintomático respecto de aquellos que afirmaron no haberlo hecho nunca, obteniendo resultados en la dirección opuesta para el grupo asintomático en todos los instrumentos de evaluación. En la misma línea, la variable "consumo diario actual de cigarrillo" arroja el mayor porcentaje en el grupo sintomático, siendo el menor para aquellos que afirmaron no hacerlo nunca.

Acorde con los resultados obtenidos en este trabajo también se encuentran los hallazgos de Anzengruber et al. (2006), ya que dejaron patente que las mujeres diagnosticadas con algún subtipo de TCA caracterizado por presentar episodios de tipo purgativo o atracones fueron las que mostraron puntaciones más altas de entre todos los subtipos de TCA incluidos en el estudio.

Aunque lo descrito por la literatura parece ir en la línea de lo encontrado en este trabajo, tenemos que mostrarnos cautos a la hora de generalizar dichos resultados. Para ello es importante considerar algunas de las limitaciones relacionadas con la validez de las conductas autodeclaradas o la naturaleza no clínica de la población incluida. En relación a la comprometida fiabilidad de las conductas autodeclaradas, esta cautela debe tenerse especialmente en cuenta para el consumo de tabaco, siendo en este caso el análisis de la cotinina la única prueba fiable y válida; prueba que por su coste e implicaciones éticas, sobre todo en población adolescente menor de edad, no se contempló. Otra prueba mucho menos costosa para validar el autoinforme del consumo de tabaco sería la medición de monóxido de carbono en aire expirado, aunque el principal inconveniente para su utilización en jóvenes son los falsos negativos en fumadores ocasionales o de pocos cigarrillos.

Otra de las limitaciones a destacar es el tipo de diseño elegido para este y otros estudios de fines parecidos cuando la muestra es no clínica, algo que no sólo compromete la validez externa, es decir, la posibilidad de generalizar los resultados obtenidos al resto de la población con similares características, sino que hace igualmente difícil poder establecer relaciones causales entre las variables objeto de estudio y los trastornos o enfermedades estudiadas. Además, junto a esta limitación, hay que tener en cuenta que la muestra está formada por 300 estudiantes y no es una muestra seleccionada de forma aleatoria; por tanto, pierde poder de generalización incluso al total de la población escolarizada con el mismo rango de edad que el utilizado en el presente estudio. Éstas son algunas de las causas por la que en este trabajo se tratan las distintas variables evaluadas como factores asociados a actitudes y comportamientos característicos de los trastornos de la conducta alimentaria y no como factores de riesgo para desarrollar anorexia, bulimia o un trastorno de la conducta alimentaria no especificado. Para poder hablar en términos de riesgo, no sólo se debe equiparar la muestra en términos de edad y género, sino también, utilizar diseños de pronóstico (cohorte), donde se pueda medir en el tiempo el efecto causal de la variable objeto de estudio en el desarrollo y/o mantenimiento de un determinado trastorno, o etiológicos (casos y controles), donde los casos respondan a una verdadera muestra clínica. Por todo ello, apuntar la necesidad de realizar en nuestro entorno estudios que nos permitan avanzar y realizar comparaciones con investigaciones realizadas previamente.

A modo de resumen, podemos concluir que en este estudio se ha podido observar que al igual que en los estudios consultados para este trabajo (Bisetto Pons et al., 2012; Marugán et al., 2010; Piran y Robinson, 2006; Stice y Shaw, 2003; Welch y Fairburn, 1998; Wiseman et al., 1997) una imagen corporal distorsionada, las preocupaciones acerca del peso así como determinadas conductas poco o nada saludables dirigidas a controlarlo o disminuirlo se relacionan positivamente con el consumo actual de tabaco y con su inicio. Algunos estudios llegan a afirmar que dentro de estas alteraciones, las relacionadas con la ingesta compulsiva/purgativa guardan aún mayor relación que aquellas dirigidas a la restricción de la comida o al excesivo ejercicio físico. A nivel general, los autores establecen esta 
relación para algunos de los síntomas relacionados con los TCA, pero no para la totalidad de criterios diagnósticos y eso es algo que se ha constatado en el presente estudio.

De una u otra forma, lo que sí se hace patente es que la prevención debe ir encaminada al cambio de los tópicos sociales actuales que sobre la figura femenina y el culto desmesurado por la delgadez aún se establecen. En esta compleja tarea deben estar implicados no sólo las estructuras sanitarias sino también las educativas, las asociaciones de usuarios, la administración política y los medios de comunicación. A menos que se trate este problema, la tendencia actual sobre la gran preocupación por el peso y la realización de dietas así como el consumo de tabaco para el control del mismo podrían continuar con las consecuentes implicaciones para la futura salud de los y las adolescentes.

\section{Reconocimientos}

La investigación se enmarca dentro del proyecto "Impulsividad, Búsqueda de Sensaciones y Consumo de Tabaco: Factores Asociados a la Anorexia y Bulimia Nerviosas", financiado por la Secretaría General de Salud Pública y Promoción de la Consejería de Salud de la Junta de Andalucía en la convocatoria de Promoción de la salud 2008.

\section{Referencias}

1. Anzengruber, D., Klump, K. L., Thornton, L., Brandt, H., Crawford, S., Fichter, M. M.,... Bulik, C. (2006). Smoking in eating disorders. Eating Behaviors, 7, 291-299. Becoña, E., Palomares, A. y García, M. P. (2000). Tabaco y salud: guía de prevención y tratamiento del tabaquismo. Madrid: Pirámide.

2. Bell, B.T., Lawton, R. y Dittmar, H. (2007). The impact of thin models in music videos on adolescent girls' body dissatisfaction. Body Image, 4(2), 137-145. https://doi.org/10.1016/j. bodyim.2007.02.003

3. Berrios-Hernández M. N., Rodríguez-Ruiz S., Pérez M., Gleaves D. H., Maysonet M. y Cepeda-Benito A. (2007). Cross-cultural assessment of eating disorders: Psychometric properties of a Spanish version of the Bulimia Test-Revised. European Eating Disorders Review, 15(6), 418-24. https://doi.org/10.1002/erv.791

4. Bisetto Pons, D., Botella Guigarro, A. y Sancho Muñoz, A. (2012). Trastornos de la conducta alimentaria y consumo de drogas en población adolescente. Adicciones, 24(1), 9-16. https://doi. org/10.20882/adicciones.112

5. $\quad$ Castro-Fornieles, J., Díaz, R., Goti, J., Calvo, R., Gonzalez, L., Serrano, L. y Gual, A. (2010). Prevalence and factors related to substance use among adolescents with eating disorders. European Addiction Research, 16(2), 61-68. https://doi.org/10.1159/000268106

6. Castro, J., Toro, J. y Salamero, M. (1991). The eating attitudes test: Validation of the Spanish version. Evaluación Psicológica, 7, 175-90.

7. Chiodo, J. (1985). The assessment of anorexia nervosa and bulimia nervosa. Progress in Behavior Modification, 19, 255-292. https://doi.org/10.1016/B978-0-12-535619-0.50011-3

8. Corral, S., González, M., Pereña, J. y Seisdedos, N. (1998). Adaptación española del Inventario de trastornos de la conducta alimentaria. EDI-2: Inventario de Trastornos de la Conducta Alimentaria. Manual. Madrid: TEA.

9. García, E., Vázquez, V., López, J. C. y Arcila, D. (2003). Validez interna y utilidad diagnóstica del Eating Disorders Inventory en mujeres mexicanas. Salud pública de México, 45, 206-210. https:// doi.org/10.1590/s0036-36342003000300010

10. Garner, D. M. (1991). Eating Disorder Inventory- 2 Professional Manual. Odessa, Florida: Psychological Assessment Resources.

11. Garner, D. M. y Garfinkel, P.E. (1979). The eating attitudes test:An index of the symptoms of anorexia nervosa. Psychological Medicine, 9, 273-279. https://doi.org/10.1017/S0033291700030762

12. Garner, D. M. y Garfinkel, P. E. (1980). Cultural Expectations of Thinnes in Woman. Psychological Reports, 47, 483-491. https://doi.org/10.2466/pr0.1980.47.2.483

13. Garner, D. M., Olmstead, M. P., Bohr, Y. y Garfinkel, P. E. (1982). The Eating attitudes test: psychometric features and clinical correlates. Psychological Medicine, 12, 871-878. https://doi. org/10.1017/S0033291700049163

14. Grupo de trabajo de la Guía de Práctica Clínica sobre Trastornos de la Conducta Alimentaria. (2009). Guía de Práctica Clínica Sobre Trastornos de La Conducta Alimentaria. Madrid: Plan de Calidad Para el Sistema Nacional de Salud del Ministerio de Sanidad y Consumo

15. Iñarritu, M. C., Cruz, V. y Morán, I. C. (2004). Instrumentos de evaluación para los trastornos de la conducta alimentaria. Revista de Salud Pública y Nutrición, 5, 1-7. 
16. King, M. B. (1989). Eating disorders in a general practice population. Prevalence, characteristics and follow-up at 12 to 18 months. Psychological Medicine, 14, 1-34. https://doi.org/10.1017/ s0264180100000515

17. King, M. B. (1991). The natural history of eating pathology in attenders to primary medical care. International Journal of Eating Disorders, 10, 379-387. https://doi.org/10.1002/1098-108X(19910 7) 10:4<379::AID-EAT2260100402>3.0.CO;2-I

18. Marugán, J., Monasterio, L. y Pavón, M. (2010). Alimentación en el adolescente. En J. D. de la SEGHNP (Ed.), Protocolos diagnóstico-terapéuticos de Gastroenterología, Hepatología y Nutrición Pediátrica (pp. 307-312). Madrid: Ergón S.A.

19. Mendoza Berjano, R., López Pérez, P. y Sagrera, M. R. (2007). Diferencias de género en la evolución del tabaquismo adolescente en España (1986-2002). Adicciones, 19(3), 273-287. https:// doi.org/10.20882/adicciones.306

20. Peláez, M. (2003). Estudio epidemiológico de los trastornos de la conducta alimentaria en población escolarizada de la comunidad de Madrid. Tesis Doctoral no publicada. Madrid: Universidad Complutense de Madrid.

21. Peláez, M. A, Labrador, F. J. y Raich R. M. (2005). Prevalencia de los trastornos de la conducta alimentaria: cuestiones metodológicas. Revista Internacional de Psicología y Terapia Psicológica, 5(2), 135-148.

22. Peláez Fernández, M. A., Raich Escursell, R. M. y Labrador Encinas, F. J. (2010). Trastornos de la conducta alimentaria en España: Revisión de estudios epidemiológicos. Revista Mexicana de Trastornos Alimentarios, 1(1), 62-75.

23. Piran, N. y Robinson, S. (2006). The association between disordered eating and substance use and abuse in women: a community-based investigation. Women \& Health, 44(1), 1-20. https:// doi.org/10.1300/J013v44n01 01

24. Rivas, T., Bersabé, R. y Castro, S. (2001). Prevalencia de los trastornos de la conducta alimentaria en adolescentes de Málaga (España). Salud Mental, 24(2), 25-31.

25. Smart, R. G., Hughes, P. H., Johnston, L. D., Anumone, A., Khant, U. y Medina Mora, M. E. (1980). Methodologie pour des enquêtes sur l'usage des drogues chez les etudiants. Ginebra: OMS.

26. Smith, M. C. y Thelen, M. H. (1984). Development and validation of a test for bulimia. Journal Consulting Clinical Psychology, 21, 167-179. https://doi.org/10.1037/0022-006x.52.5.863

27. Stice, E. y Shaw, H. (2003). Prospective relations of body image, eating and affective disturbances to smoking onset in adolescent girls: How Virginia slims. Journal of Consulting and Clinical Psychology, 71, 129-135. https://doi.org/10.1037/0022-006X.71.1.129

28. Thelen, M. H., Farmer, J., Wonderlich, D. y Smith, M. (1991). A revision of the bulimia test: the BULIT-R. Journal consulting Clinical Psychology, 3, 119-124.

29. Toro, J. (2000). La epidemiología de los trastornos de la conducta alimentaria. Medicina Clínica, 114(14), 543-544. https://doi.org/10.1016/S0025-7753(00)71357-1

30. Toro, J. y Vilardell, E. (1987). Anorexia Nerviosa. Barcelona: Martínez Roca.

31. Villalbí, J. R., Suelves, J. M., García-Continente, X., Saltó, E., Ariza, C. y Cabezas, C. (2012). Cambios en la prevalencia del tabaquismo en los adolescentes en España. Atención Primaria, 44(1), 36-42. https://doi.org/10.1016/j.aprim.2010.12.016

32. Welch, S. L. y Fairburn, C. G. (1998). Smoking and bulimia nervosa. The International Journal of Eating Disorders, 23(4), 433-437. https://doi.org/10.1002/(SICI)1098-108X(199805)23:4<433::A ID-EAT11>3.0.CO;2-X

33. Welch, G., Thompson, L. y Hall, A. (1993). The BULIT-R its realiability and clinical validity as a screening tool for DSM-III-R bulimia nervosa in a female tertiary education population. International Journal of Eating Disorder, 14, 95-105. https://doi.org/10.1002/1098-108X(199307)14:1<95:: AID-EAT2260140113>3.0.CO;2-Z

34. Williamson, D.A. (1990). Assesment of eating disorders: obesity, anorexia and bulimia nervosa. New York: Pergamon Press.

35. Wiseman, C., Turco, R., Sunday, S. y Halmi, K. (1997). Smoking and body image concerns in adolescent girls. International Journal of Eating Disorders, 24, 429-433. https://doi.org/10.1002/ (SICI)1098-108X(199812)24:4<429::AID-EAT10>3.0.CO;2-D

RECIBIDO: 08/03/2016

MODIFICADO: $15 / 09 / 2016$

ACEPTADO: 16/09/2016 